Article

\title{
Certain Notions of Neutrosophic Topological K-Algebras
}

\author{
Muhammad Akram ${ }^{1, *(D)}$, Hina Gulzar ${ }^{1}$, Florentin Smarandache ${ }^{2}$ and Said Broumi ${ }^{3}$ \\ 1 Department of Mathematics, University of the Punjab, New Campus, Lahore 54590, Pakistan; \\ hinagulzar5@gmail.com \\ 2 Department 705 Gurley Ave., University of New Mexico Mathematics \& Science, Gallup, NM 87301, USA; \\ fsmarandache@gmail.com \\ 3 Laboratory of Information Processing, Faculty of Science Ben M'Sik, University Hassan II, B.P 7955, \\ Sidi Othman, Casablanca 20000, Morocco; broumisaid78@gmail.com \\ * Correspondence: m.akram@pucit.edu.pk
}

Received: 24 September 2018 ; Accepted: 29 October 2018; Published: 30 October 2018

\begin{abstract}
The concept of neutrosophic set from philosophical point of view was first considered by Smarandache. A single-valued neutrosophic set is a subclass of the neutrosophic set from a scientific and engineering point of view and an extension of intuitionistic fuzzy sets. In this research article, we apply the notion of single-valued neutrosophic sets to $K$-algebras. We introduce the notion of single-valued neutrosophic topological $K$-algebras and investigate some of their properties. Further, we study certain properties, including $C_{5}$-connected, super connected, compact and Hausdorff, of single-valued neutrosophic topological $K$-algebras. We also investigate the image and pre-image of single-valued neutrosophic topological $K$-algebras under homomorphism.
\end{abstract}

Keywords: K-algebras; single-valued neutrosophic sets; homomorphism; compactness; $C_{5}$-connectedness

MSC: 06F35; 03G25; 03B52

\section{Introduction}

A new kind of logical algebra, known as K-algebra, was introduced by Dar and Akram in [1]. A $K$-algebra is built on a group $G$ by adjoining the induced binary operation on $G$. The group $G$ is particularly of the type in which each non-identity element is not of order 2 . This algebraic structure is, in general, non-commutative and non-associative with right identity element [1-3]. Akram et al. [4] introduced fuzzy K-algebras. They then developed fuzzy K-algebras with other researchers worldwide. The concepts and results of $K$-algebras have been broadened to the fuzzy setting frames by applying Zadeh's fuzzy set theory and its generalizations, namely, interval-valued fuzzy sets, intuitionistic fuzzy sets, interval-valued intuitionistic fuzzy sets, bipolar fuzzy sets and vague sets [5]. In handling information regarding various aspects of uncertainty, non-classical logic is considered to be a more powerful tool than the classical logic. It has become a strong mathematical tool in computer science, medical, engineering, information technology, etc. In 1998, Smarandache [6] introduced neutrosophic set as a generalization of intuitionistic fuzzy set [7]. A neutrosophic set is identified by three functions called truth-membership $(T)$, indeterminacy-membership $(I)$ and falsity-membership $(F)$ functions. To apply neutrosophic set in real-life problems more conveniently, Smarandache [6] and Wang et al. [8] defined single-valued neutrosophic sets which takes the value from the subset of $[0,1]$. Thus, a single-valued neutrosophic set is an instance of neutrosophic set.

Algebraic structures have a vital place with vast applications in various areas of life. Algebraic structures provide a mathematical modeling of related study. Neutrosophic set theory has also been 
applied to many algebraic structures. Agboola and Davazz introduced the concept of neutrosophic $B C I / B C K$-algebras and discuss elementary properties in [9]. Jun et al. introduced the notion of $(\phi, \psi)$ neutrosophic subalgebra of a $B C K / B C I$-algebra [10]. Jun et al. [11] defined interval neutrosophic sets on $B C K / B C I$-algebra [11]. Jun et al. [12] proposed neutrosophic positive implicative $N$-ideals and study their extension property [12] Several set theories and their topological structures have been introduced by many researchers to deal with uncertainties. Chang [13] was the first to introduce the notion of fuzzy topology. Later, Lowan [14], Pu and Liu [15], and Chattopadhyay and Samanta [16] introduced other concepts related to fuzzy topology. Coker [17] introduced the notion of intuitionistic fuzzy topology as a generalization of fuzzy topology. Salama and Alblowi [18] defined the topological structure of neutrosophic set theory. Akram and Dar [19] introduced the concept of fuzzy topological $K$-algebras. They extended their work on intuitionistic fuzzy topological $K$-algebras [20]. In this paper, we introduce the notion of single-valued neutrosophic topological $K$-algebras and investigate some of their properties. Further, we study certain properties, including $C_{5}$-connected, super connected, compact and Hausdorff, of single-valued neutrosophic topological $K$-algebras. We also investigate the image and pre-image of single-valued neutrosophic topological $K$-algebras under homomorphism.

\section{Preliminaries}

The notion of $K$-algebra was introduced by Dar and Akram in [1].

Definition 1. [1] Let $(G, \cdot, e)$ be a group in which each non-identity element is not of order 2. A K-algebra is a structure $\mathcal{K}=(G, \cdot \odot, e)$ over a particular group $G$, where $\odot$ is an induced binary operation $\odot: G \times G \rightarrow G$ is defined by $\odot(s, t)=s \odot t=s . t^{-1}$, and satisfy the following conditions:

(i) $(s \odot t) \odot(s \odot u)=(s \odot((e \odot u) \odot(e \odot t))) \odot s$;

(ii) $s \odot(s \odot t)=(s \odot(e \odot t) \odot s$;

(iii) $s \odot s=e$;

(iv) $s \odot e=s$; and

(v) $e \odot s=s^{-1}$

for all s, $t, u \in G$. The homomorphism between two K-algebras $\mathcal{K}_{1}$ and $\mathcal{K}_{2}$ is a mapping $f: \mathcal{K}_{1} \rightarrow \mathcal{K}_{2}$ such that, for all $u, v \in \mathcal{K}_{1}, f(u \odot v)=f(u) \odot f(v)$.

In [6], Smarandache initiated the idea of neutrosophic set theory which is a generalization of intuitionistic fuzzy set theory. Later, Smarandache and Wang et al. introduced a single-valued neutrosophic set (SNS) as an instance of neutrosophic set in [8].

Definition 2. [8] Let $Z$ be a space of points with a general element $s \in Z$. A SNS $\mathcal{A}$ in $Z$ is equipped with three membership functions: truth membership function $\left(\mathcal{T}_{\mathcal{A}}\right)$, indeterminacy membership function $\left(\mathcal{I}_{\mathcal{A}}\right)$ and falsity membership function $\left(\mathcal{F}_{\mathcal{A}}\right)$, where $\forall s \in Z, \mathcal{T}_{\mathcal{A}}(s), \mathcal{I}_{\mathcal{A}}(s), \mathcal{F}_{\mathcal{A}}(s) \in[0,1]$. There is no restriction on the sum of these three components. Therefore, $0 \leq \mathcal{T}_{\mathcal{A}}(s)+\mathcal{I}_{\mathcal{A}}(s)+\mathcal{F}_{\mathcal{A}}(s) \leq 3$.

Definition 3. [8] A single-valued neutrosophic empty set $\left(\varnothing_{S N}\right)$ and single-valued neutrosophic whole set $\left(1_{S N}\right)$ on $\mathrm{Z}$ is defined as:

- $\varnothing_{S N}(u)=\{u \in Z:(u, 0,0,1)\}$.

- $1_{S N}(u)=\{u \in Z:(u, 1,1,0)\}$.

Definition 4. [8] If $f$ is a mapping from a set $Z_{1}$ into a set $Z_{2}$, then the following statements hold:

(i) Let $\mathcal{A}$ be a SNS in $Z_{1}$ and $\mathcal{B}$ be a SNS in $Z_{2}$, then the pre-image of $\mathcal{B}$ is a SNS in $Z_{1}$, denoted by $f^{-1}(\mathcal{B})$, defined as:

$f^{-1}(\mathcal{B})=\left\{z_{1} \in Z_{1}: f^{-1}\left(\mathcal{T}_{\mathcal{B}}\right)\left(z_{1}\right)=\mathcal{T}_{\mathcal{B}}\left(f\left(z_{1}\right)\right), f^{-1}\left(\mathcal{I}_{\mathcal{B}}\right)\left(z_{1}\right)=\mathcal{I}_{\mathcal{B}}\left(f\left(z_{1}\right)\right), f^{-1}\left(\mathcal{F}_{\mathcal{B}}\right)\left(z_{1}\right)=\right.$ $\left.\mathcal{F}_{\mathcal{B}}\left(f\left(z_{1}\right)\right)\right\}$. 
(ii) Let $\mathcal{A}=\left\{z_{1} \in Z_{1}: \mathcal{T}_{\mathcal{A}}\left(z_{1}\right), \mathcal{I}_{\mathcal{A}}\left(z_{1}\right), \mathcal{F}_{\mathcal{A}}\left(z_{1}\right)\right\}$ be a SNS in $Z_{1}$ and $\mathcal{B}=\left\{z_{2} \in Z_{2}\right.$ : $\left.\mathcal{T}_{\mathcal{B}}\left(z_{2}\right), \mathcal{I}_{\mathcal{B}}\left(z_{2}\right), \mathcal{F}_{\mathcal{B}}\left(z_{2}\right)\right\}$ be a SNS in $Z_{2}$. Under the mapping $f$, the image of $\mathcal{A}$ is a SNS in $Z_{2}$, denoted by $f(\mathcal{A})$, defined as: $f(\mathcal{A})=\left\{z_{2} \in Z_{2}: f_{\text {sup }}\left(\mathcal{T}_{\mathcal{A}}\right)\left(z_{2}\right), f_{\text {sup }}\left(\mathcal{I}_{\mathcal{A}}\right)\left(z_{2}\right), f_{\text {inf }}\left(\mathcal{F}_{\mathcal{A}}\right)\left(z_{2}\right)\right\}$, where for all $z_{2} \in Z_{2}$.

$$
\begin{aligned}
& f_{\text {sup }}\left(\mathcal{T}_{\mathcal{A}}\right)\left(z_{2}\right)= \begin{cases}\sup _{z_{1} \in f^{-1}}{ }_{\left(z_{2}\right)} \mathcal{T}_{\mathcal{A}}\left(Z_{1}\right), & \text { if } f_{\left(z_{2}\right)}^{-1} \neq \varnothing, \\
0, & \text { otherwise, }\end{cases} \\
& f_{\text {sup }}\left(\mathcal{I}_{\mathcal{A}}\right)\left(z_{2}\right)= \begin{cases}\sup _{z_{1} \in f^{-1}}{\left(z_{2}\right)}_{\mathcal{I}} \mathcal{I}_{\mathcal{A}}\left(Z_{1}\right), & \text { if } f_{\left(z_{2}\right)}^{-1} \neq \varnothing, \\
0, & \text { otherwise, }\end{cases} \\
& f_{\text {inf }}\left(\mathcal{F}_{\mathcal{A}}\right)\left(z_{2}\right)= \begin{cases}\inf _{z_{1} \in f_{\left(z_{2}\right)}^{-1} \mathcal{F}_{\mathcal{A}}\left(Z_{1}\right),} & \text { if } f_{\left(z_{2}\right)}^{-1} \neq \varnothing, \\
0, & \text { otherwise. }\end{cases}
\end{aligned}
$$

We formulate the following proposition.

Proposition 1. Let $f: Z_{1} \rightarrow Z_{2}$ and $\mathcal{A},\left(\mathcal{A}_{j}, j \in J\right)$ be a SNS in $Z_{1}$ and $\mathcal{B}$ be a SNS in $Z_{2}$. Then, $f$ possesses the following properties:

(i) If $f$ is onto, then $f\left(1_{S N}\right)=1_{S N}$.

(ii) $f\left(\varnothing_{S N}\right)=\varnothing_{S N}$.

(iii) $f^{-1}\left(1_{S N}\right)=1_{S N}$.

(iv) $f^{-1}\left(\varnothing_{S N}\right)=\varnothing_{S N}$.

(v) If $f$ is onto, then $f\left(f^{-1}(\mathcal{B})=\mathcal{B}\right.$.

(vi) $f^{-1}\left(\bigcup_{i=1}^{n} \mathcal{A}_{i}\right)=\bigcup_{i=1}^{n} f^{-1}\left(\mathcal{A}_{i}\right)$

\section{Neutrosophic Topological $K$-algebras}

Definition 5. Let Z be a nonempty set. A collection $\chi$ of single-valued neutrosophic sets (SNSs) in Z is called a single-valued neutrosophic topology (SNT) on Z if the following conditions hold:

(a) $\varnothing_{S N}, 1_{S N} \in \chi$

(b) If $\mathcal{A}, \mathcal{B} \in \chi$, then $\mathcal{A} \cap \mathcal{B} \in \chi$

(c) If $\mathcal{A}_{i} \in \chi, \forall i \in I$, then $\bigcup_{i \in I} \mathcal{A}_{i} \in \chi$

The pair $(Z, \chi)$ is called a single-valued neutrosophic topological space (SNTS). Each member of $\chi$ is said to be $\chi$-open or single-valued neutrosophic open set (SNOS) and compliment of each open single-valued neutrosophic set is a single-valued neutrosophic closed set (SNCS). A discrete topology is a topology which contains all single-valued neutrosophic subsets of $Z$ and indiscrete if its elements are only $\emptyset_{S N}, 1_{S N}$.

Definition 6. Let $\mathcal{A}=\left(\mathcal{T}_{\mathcal{A}}, \mathcal{I}_{\mathcal{A}}, \mathcal{F}_{\mathcal{A}}\right)$ be a single-valued neutrosophic set in $\mathcal{K}$. Then, $\mathcal{A}$ is called a single-valued neutrosophic $K$-subalgebra of $\mathcal{K}$ if following conditions hold for $\mathcal{A}$ :

(i) $\mathcal{T}_{\mathcal{A}}(e) \geq \mathcal{T}_{\mathcal{A}}(s), \mathcal{I}_{\mathcal{A}}(e) \geq \mathcal{I}_{\mathcal{A}}(s), \mathcal{F}_{\mathcal{A}}(e) \leq \mathcal{F}_{\mathcal{A}}(s)$.

(ii) $\mathcal{T}_{\mathcal{A}}(s \odot t) \geq \min \left\{\mathcal{T}_{\mathcal{A}}(s), \mathcal{T}_{\mathcal{A}}(t)\right\}$,

$\mathcal{I}_{\mathcal{A}}(s \odot t) \geq \min \left\{\mathcal{I}_{\mathcal{A}}(s), \mathcal{I}_{\mathcal{A}}(t)\right\}$

$\mathcal{F}_{\mathcal{A}}(s \odot t) \leq \max \left\{\mathcal{F}_{\mathcal{A}}(s), \mathcal{F}_{\mathcal{A}}(t)\right\} \forall s, t \in \mathcal{K}$. 
Example 1. Consider a K-algebra $\mathcal{K}=(G, \cdot, \odot, e)$, where $G=\left\{e, x, x^{2}, x^{3}, x^{4}, x^{5}, x^{6}, x^{7}, x^{8}\right\}$ is the cyclic group of order 9 and Caley's table for $\odot$ is given as:

\begin{tabular}{c|ccccccccc}
$\odot$ & $e$ & $x$ & $x^{2}$ & $x^{3}$ & $x^{4}$ & $x^{5}$ & $x^{6}$ & $x^{7}$ & $x^{8}$ \\
\hline$e$ & $e$ & $x^{8}$ & $x^{7}$ & $x^{6}$ & $x^{5}$ & $x^{4}$ & $x^{3}$ & $x^{2}$ & $x$ \\
$x$ & $x$ & $e$ & $x^{8}$ & $x^{7}$ & $x^{6}$ & $x^{5}$ & $x^{4}$ & $x^{3}$ & $x^{2}$ \\
$x^{2}$ & $x^{2}$ & $x$ & $e$ & $x^{8}$ & $x^{7}$ & $x^{6}$ & $x^{5}$ & $x^{4}$ & $x^{3}$ \\
$x^{3}$ & $x^{3}$ & $x^{2}$ & $x$ & $e$ & $x^{8}$ & $x^{7}$ & $x^{6}$ & $x^{5}$ & $x^{4}$ \\
$x^{4}$ & $x^{4}$ & $x^{3}$ & $x^{2}$ & $x$ & $e$ & $x^{8}$ & $x^{7}$ & $x^{6}$ & $x^{5}$ \\
$x^{5}$ & $x^{5}$ & $x^{4}$ & $x^{3}$ & $x^{2}$ & $x$ & $e$ & $x^{8}$ & $x^{7}$ & $x^{6}$ \\
$x^{6}$ & $x^{6}$ & $x^{5}$ & $x^{4}$ & $x^{3}$ & $x^{2}$ & $x$ & $e$ & $x^{8}$ & $x^{7}$ \\
$x^{7}$ & $x^{7}$ & $x^{6}$ & $x^{5}$ & $x^{4}$ & $x^{3}$ & $x^{2}$ & $x$ & $e$ & $x^{8}$ \\
$x^{8}$ & $x^{8}$ & $x^{7}$ & $x^{6}$ & $x^{5}$ & $x^{4}$ & $x^{3}$ & $x^{2}$ & $x$ & $e$
\end{tabular}

If we define a single-valued neutrosophic set $\mathcal{A}, \mathcal{B}$ in $\mathcal{K}$ such that:

$$
\begin{gathered}
\mathcal{A}=\{(e, 0.4,0.5,0.8),(s, 0.3,0.4,0.7)\} \\
\mathcal{B}=\{(e, 0.3,0.4,0.8),(s, 0.2,0.3,0.6)\}
\end{gathered}
$$

$\forall s \neq e \in G$.

According to Definition 5 , the family $\left\{\varnothing_{S N}, 1_{S N}, \mathcal{A}, \mathcal{B}\right\}$ of SNSs of $K$-algebra is a SNT on $\mathcal{K}$. We define a SNS $\mathcal{A}=\left\{\mathcal{T}_{\mathcal{A}}, \mathcal{I}_{\mathcal{A}}, \mathcal{F}_{\mathcal{A}}\right\}$ in $\mathcal{K}$ such that $\mathcal{T}_{\mathcal{A}}(e)=0.7, \mathcal{I}_{\mathcal{A}}(e)=0.5, \mathcal{F}_{\mathcal{A}}(e)=0.2, \mathcal{T}_{\mathcal{A}}(s)=0.2, \mathcal{I}_{\mathcal{A}}(s)=0.4$, $\mathcal{F}_{\mathcal{A}}(s)=0.6$. Clearly, $\mathcal{A}=\left(\mathcal{T}_{\mathcal{A}}, \mathcal{I}_{\mathcal{A}}, \mathcal{F}_{\mathcal{A}}\right)$ is a SN K-subalgebra of $\mathcal{K}$.

Definition 7. Let $\mathcal{K}=(G, \cdot, \odot, e)$ be a K-algebra and let $\chi_{\mathcal{K}}$ be a topology on $\mathcal{K}$. Let $\mathcal{A}$ be a SNS in $\mathcal{K}$ and let $\chi_{\mathcal{K}}$ be a topology on $\mathcal{K}$. Then, an induced single-valued neutrosophic topology on $\mathcal{A}$ is a collection or family of single-valued neutrosophic subsets of $\mathcal{A}$ which are the intersection with $\mathcal{A}$ and single-valued neutrosophic open sets in $\mathcal{K}$ defined as $\chi_{\mathcal{A}}=\left\{\mathcal{A} \cap F: F \in \chi_{\mathcal{K}}\right\}$. Then, $\chi_{\mathcal{A}}$ is called single-valued neutrosophic induced topology on $\mathcal{A}$ or relative topology and the pair $\left(\mathcal{A}, \chi_{\mathcal{A}}\right)$ is called an induced topological space or single-valued neutrosophic subspace of $\left(\mathcal{K}, \chi_{\mathcal{K}}\right)$.

Definition 8. Let $\left(\mathcal{K}_{1}, \chi_{1}\right)$ and $\left(\mathcal{K}_{2}, \chi_{2}\right)$ be two SNTSs and let $f:\left(\mathcal{K}_{1}, \chi_{1}\right) \rightarrow\left(\mathcal{K}_{2}, \chi_{2}\right)$. Then, $f$ is called single-valued neutrosophic continuous if following conditions hold:

(i) For each SNS $\mathcal{A} \in \chi_{2}, f^{-1}(\mathcal{A}) \in \chi_{1}$.

(ii) For each $S N K$-subalgebra $\mathcal{A} \in \chi_{2}, f^{-1}(\mathcal{A})$ is a $S N K$-subalgebra $\in \chi_{1}$.

Definition 9. Let $\left(\mathcal{K}_{1}, \chi_{1}\right)$ and $\left(\mathcal{K}_{2}, \chi_{2}\right)$ be two SNTSs and let $\left(\mathcal{A}, \chi_{\mathcal{A}}\right)$ and $\left(\mathcal{B}, \chi_{\mathcal{B}}\right)$ be two single-valued neutrosophic subspaces over $\left(\mathcal{K}_{1}, \chi_{1}\right)$ and $\left(\mathcal{K}_{2}, \chi_{2}\right)$. Let $f$ be a mapping from $\left(\mathcal{K}_{1}, \chi_{1}\right)$ into $\left(\mathcal{K}_{2}, \chi_{2}\right)$, then $f$ is a mapping from $\left(\mathcal{A}, \chi_{\mathcal{A}}\right)$ to $\left(\mathcal{B}, \chi_{\mathcal{B}}\right)$ if $f(\mathcal{A}) \subset \mathcal{B}$.

Definition 10. Let $f$ be a mapping from $\left(\mathcal{A}, \chi_{\mathcal{A}}\right)$ to $\left(\mathcal{B}, \chi_{\mathcal{B}}\right)$. Then, $f$ is relatively single-valued neutrosophic continuous if for every $S N O S Y_{\mathcal{B}}$ in $\chi_{\mathcal{B}}, f^{-1}\left(Y_{\mathcal{B}}\right) \cap \mathcal{A} \in \chi_{\mathcal{A}}$.

Definition 11. Let $f$ be a mapping from $\left(\mathcal{A}, \chi_{\mathcal{A}}\right)$ to $\left(\mathcal{B}, \chi_{\mathcal{B}}\right)$. Then, $f$ is relatively single-valued neutrosophic open if for every SNOS $X_{\mathcal{A}}$ in $\chi_{\mathcal{A}}$, the image $f\left(X_{\mathcal{A}}\right) \in \chi_{\mathcal{B}}$.

Proposition 2. Let $\left(\mathcal{A}, \chi_{\mathcal{A}}\right)$ and $\left(\mathcal{B}, \chi_{\mathcal{B}}\right)$ be single-valued neutrosophic subspaces of $\left(\mathcal{K}_{1}, \chi_{1}\right)$ and $\left(\mathcal{K}_{2}, \chi_{2}\right)$, where $\mathcal{K}_{1}$ and $\mathcal{K}_{2}$ are $K$-algebras. If $f$ is a single-valued neutrosophic continuous function from $\mathcal{K}_{1}$ to $\mathcal{K}_{2}$ and $f(\mathcal{A}) \subset \mathcal{B}$. Then, $f$ is relatively single-valued neutrosophic continuous function from $\mathcal{A}$ into $\mathcal{B}$.

Definition 12. Let $\left(\mathcal{K}_{1}, \chi_{1}\right)$ and $\left(\mathcal{K}_{2}, \chi_{2}\right)$ be two SNTSs. A mapping $f:\left(\mathcal{K}_{1}, \chi_{1}\right) \rightarrow\left(\mathcal{K}_{2}, \chi_{2}\right)$ is called a single-valued neutrosophic homomorphism if following conditions hold: 
(i) $f$ is a one-one and onto function.

(ii) $f$ is a single-valued neutrosophic continuous function from $\mathcal{K}_{1}$ to $\mathcal{K}_{2}$.

(iii) $f^{-1}$ is a single-valued neutrosophic continuous function from $\mathcal{K}_{2}$ to $\mathcal{K}_{1}$.

Theorem 1. Let $\left(\mathcal{K}_{1}, \chi_{1}\right)$ be a SNTS and $\left(\mathcal{K}_{2}, \chi_{2}\right)$ be an indiscrete SNTS on $K$-algebras $\mathcal{K}_{1}$ and $\mathcal{K}_{2}$, respectively. Then, each function $f$ defined as $f:\left(\mathcal{K}_{1}, \chi_{1}\right) \rightarrow\left(\mathcal{K}_{2}, \chi_{2}\right)$ is a single-valued neutrosophic continuous function from $\mathcal{K}_{1}$ to $\mathcal{K}_{2}$. If $\left(\mathcal{K}_{1}, \chi_{1}\right)$ and $\left(\mathcal{K}_{2}, \chi_{2}\right)$ be two discrete SNTSs $\mathcal{K}_{1}$ and $\mathcal{K}_{2}$, respectively, then each homomorphism $f:\left(\mathcal{K}_{1}, \chi_{1}\right) \rightarrow\left(\mathcal{K}_{2}, \chi_{2}\right)$ is a single values neutrosophic continuous function from $\mathcal{K}_{1}$ to $\mathcal{K}_{2}$.

Proof. Let $f$ be a mapping defined as $f: \mathcal{K}_{1} \rightarrow \mathcal{K}_{2}$. Let $\chi_{1}$ be SNT on $\mathcal{K}_{1}$ and $\chi_{2}$ be SNT on $\mathcal{K}_{2}$, where $\chi_{2}=\left\{\varnothing_{S N}, 1_{S N}\right\}$. We show that $f^{-1}(\mathcal{A})$ is a single-valued neutrosophic $K$-subalgebra of $\mathcal{K}_{1}$, i.e., for each $\mathcal{A} \in \chi_{2}, f^{-1}(\mathcal{A}) \in \chi_{1}$. Since $\chi_{2}=\left\{\varnothing_{S N}, 1_{S N}\right\}$, then for any $u \in \chi_{1}$, consider $\varnothing_{S N} \in \chi_{2}$ such that $f^{-1}\left(\varnothing_{S N}\right)(u)=\varnothing_{S N}(f(u))=\varnothing_{S N}(u)$.

Therefore, $\left(f^{-1}\left(\varnothing_{S N}\right)\right)=\varnothing_{S N} \in \chi_{1}$. Likewise, $\left(f^{-1}\left(1_{S N}\right)\right)=1_{S N} \in \chi_{1}$. Hence, $f$ is a SN continuous function from $\mathcal{K}_{1}$ to $\mathcal{K}_{2}$.

Now, for the second part of the theorem, where both $\chi_{1}$ and $\chi_{2}$ are SNTSs on $\mathcal{K}_{1}$ and $\mathcal{K}_{2}$, respectively, and $f:\left(\mathcal{K}_{1}, \chi_{1}\right) \rightarrow\left(\mathcal{K}_{2}, \chi_{2}\right)$ is a homomorphism. Therefore, for all $\mathcal{A} \in \chi_{2}$ and $f^{-1} \mathcal{A} \in \chi_{1}$, where $f$ is not a usual inverse homomorphism. To prove that $f^{-1}(\mathcal{A})$ is a single-valued neutrosophic $K$-subalgebra in of $\mathcal{K}_{1}$. Let for $u, v \in \mathcal{K}_{1}$,

$$
\begin{aligned}
f^{-1}\left(\mathcal{T}_{\mathcal{A}}\right)(u \odot v)=\mathcal{T}_{\mathcal{A}}(f(u \odot v)) \\
=\mathcal{T}_{\mathcal{A}}(f(u) \odot f(v)) \\
\left.\geq \min \left\{\mathcal{T}_{\mathcal{A}}(f(u)) \odot \mathcal{T}_{(} f(v)\right)\right\} \\
=\min \left\{f^{-1}\left(\mathcal{T}_{\mathcal{A}}\right)(u), f^{-1}\left(\mathcal{T}_{\mathcal{A}}\right)(v)\right\} \\
f^{-1}\left(\mathcal{I}_{\mathcal{A}}\right)(u \odot v)=\mathcal{I}_{\mathcal{A}}(f(u \odot v)) \\
=\mathcal{I}_{\mathcal{A}}(f(u) \odot f(v)) \\
\left.\geq \min \left\{\mathcal{I}_{\mathcal{A}}(f(u)) \odot \mathcal{I}_{(} f(v)\right)\right\} \\
=\min \left\{f^{-1}\left(\mathcal{I}_{\mathcal{A}}\right)(u), f^{-1}\left(\mathcal{I}_{\mathcal{A}}\right)(v)\right\} \\
f^{-1}\left(\mathcal{F}_{\mathcal{A}}\right)(u \odot v)=\mathcal{F}_{\mathcal{A}}(f(u \odot v)) \\
=\mathcal{F}_{\mathcal{A}}(f(u) \odot f(v)) \\
\leq \max \left\{\mathcal{F}_{\mathcal{A}}(f(u)) \odot \mathcal{F}_{(f(v))\}}\left(f(v), f^{-1}\left(\mathcal{F}_{\mathcal{A}}\right)(v)\right\}\right. \\
=\max \left\{f^{-1}\left(\mathcal{F}_{\mathcal{A}}\right)(u),\right.
\end{aligned}
$$

Hence, $f$ is a single-valued neutrosophic continuous function from $\mathcal{K}_{1}$ to $\mathcal{K}_{2}$.

Proposition 3. Let $\chi_{1}$ and $\chi_{2}$ be two SNTSs on $\mathcal{K}$. Then, each homomorphism $f:\left(\mathcal{K}, \chi_{1}\right) \rightarrow\left(\mathcal{K}, \chi_{2}\right)$ is a single-valued neutrosophic continuous function.

Proof. Let $\left(\mathcal{K}, \chi_{1}\right)$ and $\left(\mathcal{K}, \chi_{2}\right)$ be two SNTSs, where $\mathcal{K}$ is a $K$-algebra. To prove the above result, it is enough to show that result is false for a particular topology. Let $\mathcal{A}=\left(\mathcal{T}_{\mathcal{A}}, \mathcal{I}_{\mathcal{A}}, \mathcal{F}_{\mathcal{A}},\right)$ and $\mathcal{B}=\left(\mathcal{T}_{\mathcal{B}}, \mathcal{I}_{\mathcal{B}}, \mathcal{F}_{\mathcal{B}}\right)$ be two SNSs in $\mathcal{K}$. Take $\chi_{1}=\left\{\varnothing_{S N}, 1_{S N}, \mathcal{A}\right\}$ and $\chi_{2}=\left\{\varnothing_{S N}, 1_{S N}, \mathcal{B}\right\}$. If $f$ : $\left(\mathcal{K}, \chi_{1}\right) \rightarrow\left(\mathcal{K}, \chi_{2}\right)$, defined by $f(u)=e \odot u$, for all $u \in \mathcal{K}$, then $f$ is a homomorphism. Now, for $u \in \mathcal{A}, v \in \chi_{2},\left(f^{-1}(\mathcal{B})\right)(u)=\mathcal{B}(f(u))=\mathcal{B}(e \odot u)=\mathcal{B}(u), \forall u \in \mathcal{K}$, i.e., $f^{-1}(\mathcal{B})=\mathcal{B}$. Therefore, $\left(f^{-1}(\mathcal{B})\right) \notin \chi_{1}$. Hence, $f$ is not a single-valued neutrosophic continuous mapping.

Definition 13. Let $\mathcal{K}=(G, \cdot \odot, e)$ be a $K$-algebra and $\chi$ be a SNT on $\mathcal{K}$. Let $\mathcal{A}$ be a single-valued neutrosophic K-algebra (K-subalgebra) of $\mathcal{K}$ and $\chi_{\mathcal{A}}$ be a SNT on $\mathcal{A}$. Then, $\mathcal{A}$ is said to be a single-valued neutrosophic topological K-algebra (K-subalgebra) on $\mathcal{K}$ if the self mapping $\rho_{a}:\left(\mathcal{A}, \chi_{\mathcal{A}}\right) \rightarrow\left(\mathcal{A}, \chi_{\mathcal{A}}\right)$ defined as $\rho_{a}(u)=u \odot a, \forall a \in \mathcal{K}$, is a relatively single-valued neutrosophic continuous mapping. 
Theorem 2. Let $\chi_{1}$ and $\chi_{2}$ be two SNTSs on $\mathcal{K}_{1}$ and $\mathcal{K}_{2}$, respectively, and $f: \mathcal{K}_{1} \rightarrow \mathcal{K}_{2}$ be a homomorphism such that $f^{-1}\left(\chi_{2}\right)=\chi_{1}$. If $\mathcal{A}=\left\{\mathcal{T}_{\mathcal{A}}, \mathcal{I}_{\mathcal{A}}, \mathcal{F}_{\mathcal{A}}\right\}$ is a single-valued neutrosophic topological K-algebra of $\mathcal{K}_{2}$, then $f^{-1}(\mathcal{A})$ is a single-valued neutrosophic topological K-algebra of $\mathcal{K}_{1}$.

Proof. Let $\mathcal{A}=\left\{\mathcal{T}_{\mathcal{A}}, \mathcal{I}_{\mathcal{A}}, \mathcal{F}_{\mathcal{A}}\right\}$ be a single-valued neutrosophic topological $K$-algebra of $\mathcal{K}_{2}$. To prove that $f^{-1}(\mathcal{A})$ be a single-valued neutrosophic topological $K$-algebra of $\mathcal{K}_{1}$. Let for any $u, v \in \mathcal{K}_{1}$,

$$
\begin{aligned}
& \mathcal{T}_{f^{-1}(\mathcal{A})}(u \odot v)=\mathcal{T}_{\mathcal{A}}(f(u \odot v)) \geq \min \left\{\mathcal{T}_{\mathcal{A}}(f(u)), \mathcal{T}_{\mathcal{A}}(f(v))\right\} \\
&=\min \left\{\mathcal{T}_{f^{-1}(\mathcal{A})}(u), \mathcal{T}_{f^{-1}(\mathcal{A})}(v)\right\} \\
& \mathcal{I}_{f^{-1}(\mathcal{A})}(u \odot v)=\mathcal{I}_{\mathcal{A}}(f(u \odot v)) \\
& \geq \min \left\{\mathcal{I}_{\mathcal{A}}(f(u)), \mathcal{I}_{\mathcal{A}}(f(v))\right\} \\
& \quad=\min \left\{\mathcal{I}_{f^{-1}(\mathcal{A})}(u), \mathcal{I}_{f^{-1}(\mathcal{A})}(v)\right\} \\
& \mathcal{F}_{f^{-1}(\mathcal{A})}(u \odot v)=\mathcal{F}_{\mathcal{A}}(f(u \odot v)) \\
& \leq \max \left\{\mathcal{F}_{\mathcal{A}}(f(u)), \mathcal{F}_{\mathcal{A}}(f(v))\right\} \\
&=\max \left\{\mathcal{F}_{f^{-1}(\mathcal{A})}(u), \mathcal{F}_{f^{-1}(\mathcal{A})}(v)\right\}
\end{aligned}
$$

Hence, $f^{-1}(\mathcal{A})$ is a single-valued neutrosophic $K$-algebra of $\mathcal{K}_{1}$.

Now, we prove that $f^{-1}(\mathcal{A})$ is single-valued neutrosophic topological $K$-algebra of $\mathcal{K}_{1}$. Since $f$ is a single-valued neutrosophic continuous function, then by proposition 3.1, $f$ is also a relatively single-valued neutrosophic continuous function which maps $\left(f^{-1}(\mathcal{A}), \chi_{f^{-1}(\mathcal{A})}\right)$ to $\left(\mathcal{A}, \chi_{\mathcal{A}}\right)$.

Let $a \in \mathcal{K}_{1}$ and $Y$ be a SNS in $\chi_{\mathcal{A}}$, and let $X$ be a SNS in $\chi_{f^{-1}(\mathcal{A})}$ such that

$$
f^{-1}(Y)=X
$$

We are to prove that $\rho_{a}:\left(f^{-1}(\mathcal{A}), \chi_{f^{-1}(\mathcal{A})}\right) \rightarrow\left(f^{-1}(\mathcal{A}), \chi_{f^{-1}(\mathcal{A})}\right)$ is relatively single-valued neutrosophic continuous mapping, then for any $a \in \mathcal{K}_{1}$, we have

$$
\begin{aligned}
\mathcal{T}_{\rho_{a}^{-1}(X)}(u)=\mathcal{T}_{(X)}\left(\rho_{a}(u)\right)=\mathcal{T}_{(X)}(u \odot a) \\
=\mathcal{T}_{f}^{-1}(Y)(u \odot a)=\mathcal{T}_{(Y)}(f(u \odot a)) \\
=\mathcal{T}_{(Y)}(f(u) \odot f(a))=\mathcal{T}_{(Y)}\left(\rho_{f(a)}(f(u))\right) \\
=\mathcal{T}_{\rho^{-1} f(a) Y}(f(u))=\mathcal{T}_{f^{-1}\left(\rho_{f(a)}^{-1}(Y)(u)\right),} \\
\mathcal{I}_{\rho_{a}^{-1}(X)}(u)=\mathcal{I}_{(X)}\left(\rho_{a}(u)\right)=\mathcal{I}_{(X)}(u \odot a) \\
=\mathcal{I}_{f-1}(Y)(u \odot a)=\mathcal{I}_{(Y)}(f(u \odot a)) \\
=\mathcal{I}_{(Y)}(f(u) \odot f(a))=\mathcal{I}_{(Y)}\left(\rho_{f(a)}(f(u))\right) \\
=\mathcal{I}_{\rho^{-1} f(a) Y}(f(u))=\mathcal{I}_{f^{-1}}\left(\rho_{f(a)}^{-1}(Y)(u)\right), \\
\mathcal{F}_{\rho_{a}^{-1}(X)}(u)=\mathcal{F}_{(X)}\left(\rho_{a}(u)\right)=\mathcal{F}_{(X)}(u \odot a) \\
=\mathcal{F}_{f^{-1}(Y)}(u \odot a)=\mathcal{F}_{(Y)}(f(u \odot a)) \\
=\mathcal{F}_{(Y)}(f(u) \odot f(a))=\mathcal{F}_{(Y)}\left(\rho_{f(a)}(f(u))\right) \\
=\mathcal{F}_{\rho^{-1} f(a) Y}(f(u))=\mathcal{F}_{f^{-1}}\left(\rho_{f(a)}^{-1}(Y)(u)\right) .
\end{aligned}
$$

It concludes that $\rho_{a}^{-1}(X)=f^{-1}\left(\rho_{f(a)}^{-1}(Y)\right)$. Thus, $\rho_{a}^{-1}(X) \cap f^{-1}(\mathcal{A})=f^{-1}\left(\rho_{f(a)}^{-1}(Y)\right) \cap f^{-1}(\mathcal{A})$ is a SNS in $f^{-1}(\mathcal{A})$ and a SNS in $\chi_{f^{-1}(\mathcal{A})}$. Hence, $f^{-1}(\mathcal{A})$ and a single-valued neutrosophic topological $\mathrm{K}$-algebra of $\mathcal{K}$. Hence, the proof.

Theorem 3. Let $\left(\mathcal{K}_{1}, \chi_{1}\right)$ and $\left(\mathcal{K}_{2}, \chi_{2}\right)$ be two SNTSs on $\mathcal{K}_{1}$ and $\mathcal{K}_{2}$, respectively, and let $f$ be a bijective homomorphism of $\mathcal{K}_{1}$ into $\mathcal{K}_{2}$ such that $f\left(\chi_{1}\right)=\chi_{2}$. If $\mathcal{A}$ is a single-valued neutrosophic topological K-algebra of $\mathcal{K}_{1}$, then $f(\mathcal{A})$ is a single-valued neutrosophic topological K-algebra of $\mathcal{K}_{2}$.

Proof. Suppose that $\mathcal{A}=\left\{\mathcal{T}_{\mathcal{A}}, \mathcal{I}_{\mathcal{A}}, \mathcal{F}_{\mathcal{A}}\right\}$ is a SN topological $K$-algebra of $\mathcal{K}_{1}$. To prove that $f(\mathcal{A})$ is a single-valued neutrosophic topological $K$-algebra of $\mathcal{K}_{2}$, let, for $u, v \in \mathcal{K}_{2}$, 


$$
f(\mathcal{A})=\left(f_{\text {sup }}\left(\mathcal{T}_{\mathcal{A}}\right)(v), f_{\text {sup }}\left(\mathcal{I}_{\mathcal{A}}\right)(v), f_{\text {inf }}\left(\mathcal{F}_{\mathcal{A}}\right)(v)\right)
$$

Let $a_{o} \in f^{-1}(u), b_{o} \in f^{-1}(v)$ such that

$$
\begin{aligned}
& \sup _{x \in f^{-1}(u)} \mathcal{T}_{\mathcal{A}}(x)=\mathcal{T}_{\mathcal{A}}\left(a_{0}\right), \sup _{x \in f^{-1}(v)} \mathcal{T}_{\mathcal{A}}(x)=\mathcal{T}_{\mathcal{A}}\left(b_{o}\right), \\
& \sup _{x \in f^{-1}(u)} \mathcal{I}_{\mathcal{A}}(x)=\mathcal{I}_{\mathcal{A}}\left(a_{o}\right), \sup _{x \in f^{-1}(v)} \mathcal{I}_{\mathcal{A}}(x)=\mathcal{I}_{\mathcal{A}}\left(b_{o}\right), \\
& \inf _{x \in f^{-1}(u)} \mathcal{F}_{\mathcal{A}}(x)=\mathcal{F}_{\mathcal{A}}\left(a_{o}\right), \inf _{x \in f^{-1}(v)} \mathcal{F}_{\mathcal{A}}(x)=\mathcal{F}_{\mathcal{A}}\left(b_{o}\right) .
\end{aligned}
$$

Now,

$$
\begin{aligned}
\mathcal{T}_{f(\mathcal{A})}(u \odot v) & =\sup _{x \in f^{-1}(u \odot v)} \mathcal{T}_{\mathcal{A}}(x) \\
& \geq \mathcal{T}_{\mathcal{A}}\left(a_{o}, b_{0}\right) \\
& \geq \min \left\{\mathcal{T}_{\mathcal{A}}\left(a_{0}\right), \mathcal{T}_{\mathcal{A}}\left(b_{0}\right)\right\} \\
& =\min \left\{\sup _{x \in f^{-1}(u)} \mathcal{T}_{\mathcal{A}}(x), \sup _{x \in f^{-1}(v)} \mathcal{T}_{\mathcal{A}}(x)\right\} \\
& =\min \left\{\mathcal{T}_{f(\mathcal{A})}(u), \mathcal{T}_{f(\mathcal{A})}(v)\right\}, \\
\mathcal{I}_{f(\mathcal{A})}(u \odot v) & =\sup _{x \in f^{-1}(u \odot v)} \mathcal{I}_{\mathcal{A}}(x) \\
& \geq \mathcal{I}_{\mathcal{A}}\left(a_{o}, b_{0}\right) \\
& \geq \min \left\{\mathcal{I}_{\mathcal{A}}\left(a_{0}\right), \mathcal{I}_{\mathcal{A}}\left(b_{o}\right)\right\} \\
& =\min \left\{\sup _{x \in f^{-1}(u)} \mathcal{I}_{\mathcal{A}}(x), \sup _{x \in f^{-1}(v)} \mathcal{I}_{\mathcal{A}}(x)\right\} \\
& =\min \left\{\mathcal{I}_{f(\mathcal{A})}(u), \mathcal{I}_{f(\mathcal{A})}(v)\right\}, \\
\mathcal{F}_{f(\mathcal{A})}(u \odot v) & =\inf _{x \in f^{-1}(u \odot v)} \mathcal{F}_{\mathcal{A}}(x) \\
& \leq \mathcal{F}_{\mathcal{A}}\left(a_{o}, b_{o}\right) \\
& \leq \max \left\{\mathcal{F}_{\mathcal{A}}\left(a_{o}\right), \mathcal{F}_{\mathcal{A}}\left(b_{o}\right)\right\} \\
& =\max \left\{\inf _{x \in f^{-1}(u)} \mathcal{F}_{\mathcal{A}}(x), \inf _{x \in f^{-1}(v)} \mathcal{F}_{\mathcal{A}}(x)\right\} \\
& =\max \left\{\mathcal{F}_{f(\mathcal{A})}(u), \mathcal{F}_{f(\mathcal{A})}(v)\right\} .
\end{aligned}
$$

Hence, $f(\mathcal{A})$ is a single-valued neutrosophic $K$-subalgebra of $\mathcal{K}_{2}$. Now, we prove that the self mapping $\rho_{b}:\left(f(\mathcal{A}), \chi_{f}(\mathcal{A})\right) \rightarrow\left(f(\mathcal{A}), \chi_{f}(\mathcal{A})\right)$, defined by $\rho_{b}(v)=v \odot b$, for all $b \in \mathcal{K}_{2}$, is a relatively single-valued neutrosophic continuous mapping. Let $Y_{\mathcal{A}}$ be a SNS in $\chi_{\mathcal{A}}$, there exists a SNS " $Y^{\text {" in }} \chi_{1}$

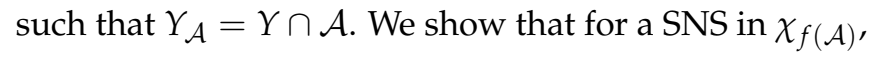

$$
\rho_{b}^{-1}\left(Y_{f(\mathcal{A})}\right) \cap f(\mathcal{A}) \in \chi_{f(\mathcal{A})}
$$

Since $f$ is an injective mapping, then $f\left(Y_{\mathcal{A}}\right)=f(Y \cap \mathcal{A})=f(Y) \cap f(\mathcal{A})$ is a SNS in $\chi_{f(\mathcal{A})}$ which shows that $f$ is relatively single-valued neutrosophic open. In addition, $f$ is surjective, then for all $b \in \mathcal{K}_{2}, a=f(b)$, where $a \in \mathcal{K}_{1}$. 
Now,

$$
\begin{aligned}
& \mathcal{T}_{f^{-1}\left(\rho^{-1}{ }_{b}\left(Y_{f(\mathcal{A})}\right)\right)}(u)=\mathcal{T}_{f^{-1}\left(\rho^{-1}{ }_{f}(a)\left(Y_{f(\mathcal{A})}\right)\right)}(u) \\
& =\mathcal{T}_{\rho^{-1} f(a)\left(Y_{f(\mathcal{A})}\right)}(f(u)) \\
& =\mathcal{T}_{\left(Y_{f(\mathcal{A})}\right)}\left(\rho_{f(a)}(f(u))\right) \\
& =\mathcal{T}_{\left(Y_{f(\mathcal{A})}\right)}(f(u) \odot f(a)) \\
& =\mathcal{T}_{f^{-1}\left(Y_{f(\mathcal{A})}\right)}(u \odot a) \\
& =\mathcal{T}_{f^{-1}\left(Y_{f(\mathcal{A})}\right)}\left(\rho_{a}(u)\right) \\
& =\mathcal{T}_{\rho^{-1}(a)}\left(f^{-1}\left(Y_{f(\mathcal{A})}\right)\right)(u), \\
& \mathcal{I}_{f^{-1}\left(\rho^{-1}{ }_{b}\left(Y_{f(\mathcal{A})}\right)\right)}(u)=\mathcal{I}_{f^{-1}\left(\rho^{-1} f(a)\left(Y_{f(\mathcal{A})}\right)\right)}(u) \\
& =\mathcal{I}_{\rho^{-1} f(a)\left(Y_{f(\mathcal{A})}\right)}(f(u)) \\
& =\mathcal{I}_{\left(Y_{f(\mathcal{A})}\right)}\left(\rho_{f(a)}(f(u))\right) \\
& =\mathcal{I}_{\left(Y_{f(\mathcal{A})}\right)}(f(u) \odot f(a)) \\
& =\mathcal{I}_{f^{-1}\left(Y_{f(\mathcal{A})}\right)}(u \odot a) \\
& =\mathcal{I}_{f^{-1}\left(Y_{f(\mathcal{A})}\right)}\left(\rho_{a}(u)\right) \\
& =\mathcal{I}_{\rho^{-1}(a)}\left(f^{-1}\left(Y_{f(\mathcal{A})}\right)\right)(u) \text {, } \\
& \mathcal{F}_{f^{-1}\left(\rho^{-1} b\left(Y_{f(\mathcal{A})}\right)\right)}(u)=\mathcal{F}_{f^{-1}\left(\rho^{-1}{ }_{f}(a)\left(Y_{f(\mathcal{A})}\right)\right)}(u) \\
& =\mathcal{F}_{\rho^{-1}{ }_{f}(a)\left(Y_{f(\mathcal{A})}\right)}(f(u)) \\
& =\mathcal{F}_{\left(Y_{f(\mathcal{A})}\right)}\left(\rho_{f(a)}(f(u))\right) \\
& =\mathcal{F}_{\left(Y_{f(\mathcal{A})}\right)}(f(u) \odot f(a)) \\
& =\mathcal{F}_{f^{-1}\left(Y_{f(\mathcal{A})}\right)}(u \odot a) \\
& =\mathcal{F}_{f^{-1}\left(Y_{f(\mathcal{A})}\right)}\left(\rho_{a}(u)\right) \\
& =\mathcal{F}_{\rho^{-1}(a)}\left(f^{-1}\left(Y_{f(\mathcal{A})}\right)\right)(u) \text {. }
\end{aligned}
$$

This implies that $f^{-1}\left(\rho_{(b)}^{-1}\left(\left(Y_{f(\mathcal{A})}\right)\right)\right)=\rho_{(a)}^{-1}\left(f^{-1}\left(Y_{(\mathcal{A})}\right)\right)$. Since $\rho_{a}:\left(\mathcal{A}, \chi_{\mathcal{A}}\right) \rightarrow\left(\mathcal{A}, \chi_{\mathcal{A}}\right)$ is relatively single-valued neutrosophic continuous mapping and $f$ is relatively single-valued neutrosophic continues mapping from $\left(\mathcal{A}, \chi_{\mathcal{A}}\right)$ into $\left(f(\mathcal{A}), \chi_{f(\mathcal{A})}\right), f^{-1}\left(\rho_{(b)}^{-1}\left(\left(Y_{f(\mathcal{A})}\right)\right)\right) \cap \mathcal{A}=\rho_{(a)}^{-1}\left(f^{-1}\left(Y_{(\mathcal{A})}\right)\right) \cap \mathcal{A}$ is a SNS in $\chi_{\mathcal{A}}$. Hence, $f\left(f^{-1}\left(\rho_{(b)}\left(\left(Y_{f(\mathcal{A})}\right)\right)\right) \cap \mathcal{A}\right)=\rho_{(b)}^{-1}\left(Y_{f(\mathcal{A})}\right) \cap f(\mathcal{A})$ is a SNS in $\chi_{\mathcal{A}}$, which completes the proof.

Example 2. Let $\mathcal{K}=(G, \cdot \odot, e)$ be a K-algebra, where $G=\left\{e, x, x^{2}, x^{3}, x^{4}, x^{5}, x^{6}, x^{7}, x^{8}\right\}$ is the cyclic group of order 9 and Caley's table for $\odot$ is given in Example 1. We define a SNS as:

$$
\begin{aligned}
& \mathcal{A}=\{(e, 0.4,0.5,0.8),(s, 0.3,0.4,0.6)\}, \\
& \mathcal{B}=\{(e, 0.3,0.4,0.8),(s, 0.2,0.3,0.6)\}
\end{aligned}
$$

for all $s \neq e \in G$, where $\mathcal{A}, \mathcal{B} \in[0,1]$. The collection $\chi_{\mathcal{K}}=\left\{\varnothing_{S N}, 1_{S N}, \mathcal{A}, \mathcal{B}\right\}$ of SNSs of $\mathcal{K}$ is a SNT on $\mathcal{K}$ and $\left(\mathcal{K}, \chi_{\mathcal{K}}\right)$ is a SNTS. Let $\mathcal{C}$ be a SNS in $\mathcal{K}$, defined as:

$$
\mathcal{C}=\{(e, 0.7,0.5,0.2),(s, 0.5,0.4,0.6)\}, \forall s \neq e \in G .
$$


Clearly, $\mathcal{C}$ is a single-valued neutrosophic K-subalgebra of $\mathcal{K}$. By direct calculations relative topology $\chi_{\mathcal{C}}$ is obtained as $\chi_{\mathcal{C}}=\left\{\varnothing_{\mathcal{A}}, 1_{\mathcal{A}}, \mathcal{A}\right\}$. Then, the pair $\left(\mathcal{C}, \chi_{\mathcal{C}}\right)$ is a single-valued neutrosophic subspace of $\left(\mathcal{K}, \chi_{\mathcal{K}}\right)$. We show that $\mathcal{C}$ is a single-valued neutrosophic topological $K$-subalgebra of $\mathcal{K}$, i.e., the self mapping $\rho_{a}:\left(\mathcal{C}, \chi_{\mathcal{C}}\right) \rightarrow\left(\mathcal{C}, \chi_{\mathcal{C}}\right)$ defined by $\rho_{a}(u)=u \odot a, \forall a \in \mathcal{K}$ is relatively single-valued neutrosophic continuous mapping, i.e., for a SNOS $\mathcal{A}$ in $\left(\mathcal{C}, \chi_{\mathcal{C}}\right), \rho_{a}^{-1}(\mathcal{A}) \cap \mathcal{C} \in \chi_{\mathcal{C}}$. Since $\rho_{a}$ is homomorphism, then $\rho_{a}^{-1}(\mathcal{A}) \cap \mathcal{C}=\mathcal{A} \in \chi_{\mathcal{C}}$. Therefore, $\rho_{a}:\left(\mathcal{C}, \chi_{\mathcal{C}}\right) \rightarrow\left(\mathcal{C}, \chi_{\mathcal{C}}\right)$ is relatively single-valued neutrosophic continuous mapping. Hence, $\mathcal{C}$ is a single-valued neutrosophic topological K-algebra of $\mathcal{K}$.

Example 3. Let $\mathcal{K}=(G, \cdot \odot, e)$ be a K-algebra, where $G=\left\{e, x, x^{2}, x^{3}, x^{4}, x^{5}, x^{6}, x^{7}, x^{8}\right\}$ is the cyclic group of order 9 and Caley's table for $\odot$ is given in Example 3.1. We define a SNS as:

$$
\begin{aligned}
& \mathcal{A}=\{(e, 0.4,0.5,0.8),(s, 0.3,0.4,0.6)\}, \\
& \mathcal{B}=\{(e, 0.3,0.4,0.8),(s, 0.2,0.3,0.6)\}, \\
& \mathcal{D}=\{(e, 0.2,0.1,0.3),(s, 0.1,0.1,0.5)\},
\end{aligned}
$$

for all $s \neq e \in G$, where $\mathcal{A}, \mathcal{B} \in[0,1]$. The collection $\chi_{1}=\left\{\varnothing_{S N}, 1_{S N}, \mathcal{D}\right\}$ and $\chi_{2}=\left\{\varnothing_{S N}, 1_{S N}, \mathcal{A}, \mathcal{B}\right\}$ of SNSs of $\mathcal{K}$ are $S N T$ s on $\mathcal{K}$ and $\left(\mathcal{K}, \chi_{1}\right),\left(\mathcal{K}, \chi_{2}\right)$ be two SNTSs. Let $\mathcal{C}$ be a SNS in $\left(\mathcal{K}, \chi_{2}\right)$, defined as:

$$
\mathcal{C}=\{(e, 0.7,0.5,0.2),(s, 0.5,0.4,0.6)\}, \forall s \neq e \in G .
$$

Now, Let $f:\left(\mathcal{K}, \chi_{1}\right) \rightarrow\left(\mathcal{K}, \chi_{2}\right)$ be a homomorphism such that $f^{-1}\left(\chi_{2}\right)=\chi_{1}$ (we have not consider $\mathcal{K}$ to be distinct), then, by Proposition $3, f$ is a single-valued neutrosophic continuous function and $f$ is also relatively single-valued neutrosophic continues mapping from $\left(\mathcal{K}, \chi_{1}\right)$ into $\left(\mathcal{K}, \chi_{2}\right)$. Since $\mathcal{C}$ is a SNS in $\left(\mathcal{K}, \chi_{2}\right)$ and with relative topology $\chi_{\mathcal{C}}=\left\{\varnothing_{\mathcal{A}}, 1_{\mathcal{A}}, \mathcal{A}\right\}$ is also a single-valued neutrosophic topological K-algebra of $\left(\mathcal{K}, \chi_{2}\right)$. We prove that $f^{-1}(\mathcal{C})$ is a single-valued neutrosophic topological $K$-algebra in $\left(\mathcal{K}, \chi_{1}\right)$. Since $f$ is a continuous function, then, by Definition $8, f^{-1}(\mathcal{C})$ is a single-valued neutrosophic $K$-subalgebra in $\left(\mathcal{K}, \chi_{1}\right)$. To prove that $f^{-1}(c)$ is a single-valued neutrosophic topological K-algebra, then for $b \in \mathcal{K}_{1}$ take

$$
\rho_{b}:\left(f^{-1}(\mathcal{C}), \chi_{f^{-1}(\mathcal{C})}\right) \rightarrow\left(f^{-1}(\mathcal{C}), \chi_{f^{-1}(\mathcal{C})}\right),
$$

for $\mathcal{A} \in \chi_{f^{-1}(C)}, \rho_{b}^{-1}(\mathcal{A}) \cap f^{-1}(\mathcal{C}) \in \chi_{f^{-1}(C)}$ which shows that $f^{-1}(C)$ is a single-valued neutrosophic topological K-algebra in $\left(\mathcal{K}, \chi_{1}\right)$. Similarly, we can show that $f(\mathcal{C})$ is a a single-valued neutrosophic topological $K$-algebra in $\left(\mathcal{K}, \chi_{2}\right)$ by considering a bijective homomorphism.

Definition 14. Let $\chi$ be a SNT on $\mathcal{K}$ and $(\mathcal{K}, \chi)$ be a SNTS. Then, $(\mathcal{K}, \chi)$ is called single-valued neutrosophic $C_{5}$-disconnected topological space if there exist a SNOS and SNCS $\mathcal{H}$ such that $\mathcal{H}=\left(\mathcal{T}_{\mathcal{H}}, \mathcal{I}_{\mathcal{H}}, \mathcal{F}_{\mathcal{H}},\right) \neq 1_{S N}$ and $\mathcal{H}=\left(\mathcal{T}_{\mathcal{H}}, \mathcal{I}_{\mathcal{H}}, \mathcal{F}_{\mathcal{H}}\right) \neq \varnothing_{S N}$, otherwise $(\mathcal{K}, \chi)$ is called single-valued neutrosophic $C_{5}$-connected.

Example 4. Every indiscrete SNT space on $\mathcal{K}$ is $C_{5}$-connected.

Proposition 4. Let $\left(\mathcal{K}_{1}, \chi_{1}\right)$ and $\left(\mathcal{K}_{2}, \chi_{2}\right)$ be two SNTSs and $f:\left(\mathcal{K}_{1}, \chi_{1}\right) \rightarrow\left(\mathcal{K}_{2}, \chi_{2}\right)$ be a surjective single-valued neutrosophic continuous mapping. If $\left(\mathcal{K}_{1}, \chi_{1}\right)$ is a single-valued neutrosophic $C_{5}$-connected space, then $\left(\mathcal{K}_{2}, \chi_{2}\right)$ is also a single-valued neutrosophic $C_{5}$-connected space.

Proof. Suppose on contrary that $\left(\mathcal{K}_{2}, \chi_{2}\right)$ is a single-valued neutrosophic $C_{5}$-disconnected space. Then, by Definition 14 , there exist both SNOS and SNCS $\mathcal{H}$ be such that $\mathcal{H} \neq 1_{S N}$ and $\mathcal{H} \neq \varnothing_{S N}$. Since $f$ is a single-valued neutrosophic continuous and onto function, so $f^{-1}(\mathcal{H})=1_{S N}$ or $f^{-1}(\mathcal{H})=\varnothing_{S N}$, where $f^{-1}(\mathcal{H})$ is both SNOS and SNCS. Therefore,

$$
\mathcal{H}=f\left(f^{-1}(\mathcal{H})\right)=f\left(1_{S N}\right)=1_{S N}
$$

and

$$
\mathcal{H}=f\left(f^{-1}(\mathcal{H})\right)=f\left(\varnothing_{S N}\right)=\varnothing_{S N},
$$


a contradiction. Hence, $\left(\mathcal{K}_{2}, \chi_{2}\right)$ is a single-valued neutrosophic $C_{5}$-connected space.

Corollary 1. Let $\chi$ be a SNT on $\mathcal{K}$. Then, $(\mathcal{K}, \chi)$ is called a single-valued neutrosophic $C_{5}$-connected space if and only if there does not exist a single-valued neutrosophic continuous map $f:(\mathcal{K}, \chi) \rightarrow\left(\mathcal{F}_{T}, \chi_{T}\right)$ such that $f \neq 1_{S N}$ and $f \neq \varnothing_{S N}$

Definition 15. Let $\mathcal{A}=\left\{\mathcal{T}_{\mathcal{A}}, \mathcal{I}_{\mathcal{A}}, \mathcal{F}_{\mathcal{A}}\right\}$ be a SNS in $\mathcal{K}$. Let $\chi$ be a SNT on $\mathcal{K}$. The interior and closure of $\mathcal{A}$ in $\mathcal{K}$ is defined as:

$\mathcal{A}^{\text {Int }}$ : The union of SNOSs which contained in $\mathcal{A}$.

$\mathcal{A}^{\text {Clo }}$ : The intersection of SNCSs for which $\mathcal{A}$ is a subset of these SNCSs.

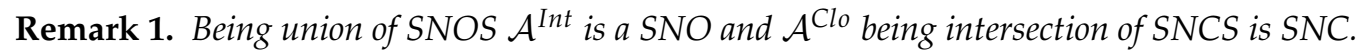

Theorem 4. Let $\mathcal{A}$ be a SNS in a SNTS $(\mathcal{K}, \chi)$. Then, $\mathcal{A}^{\text {Int }}$ is such an open set which is the largest open set of $\mathcal{K}$ contained in $\mathcal{A}$.

Corollary 2. $\mathcal{A}=\left(\mathcal{T}_{\mathcal{A}}, \mathcal{I}_{\mathcal{A}}, \mathcal{F}_{\mathcal{A}}\right)$ is a SNOS in $\mathcal{K}$ if and only if $\mathcal{A}^{\text {Int }}=\mathcal{A}$ and $\mathcal{A}=\left(\mathcal{T}_{\mathcal{A}}, \mathcal{I}_{\mathcal{A}}, \mathcal{F}_{\mathcal{A}}\right)$ is a SNCS in $\mathcal{K}$ if and only if $\mathcal{A}^{\text {Clo }}=\mathcal{A}$.

Proposition 5. Let $\mathcal{A}$ be a SNS in $\mathcal{K}$. Then, following results hold for $\mathcal{A}$ :

(i) $\left(1_{S N}\right)^{\text {Int }}=1_{S N}$.

(ii) $\left(\varnothing_{S N}\right)^{C l o}=\varnothing_{S N}$.

(iii) $\overline{(\mathcal{A})}^{\text {Int }}=\overline{(\mathcal{A})^{\mathrm{Clo}}}$.

(iv) $\overline{(\mathcal{A})}^{\mathrm{Clo}}=\overline{(\mathcal{A})^{\text {Int }}}$.

Definition 16. Let $\mathcal{K}$ be a K-algebra and $\chi$ be a SNT on $\mathcal{K} . A S N O S \mathcal{A}$ in $\mathcal{K}$ is said to be single-valued neutrosophic regular open if

$$
\mathcal{A}=\left(\mathcal{A}^{\text {Clo }}\right)^{\mathrm{Int}}
$$

Remark 2. Every SNOS which is regular is single-valued neutrosophic open and every single-valued neutrosophic closed and open set is a single-valued neutrosophic regular open.

Definition 17. A single-valued neutrosophic super connected K-algebra is such a K-algebra in which there does not exist a single-valued neutrosophic regular open set $\mathcal{A}=\left(\mathcal{T}_{\mathcal{A}}, \mathcal{I}_{\mathcal{A}}, \mathcal{F}_{\mathcal{A}}\right)$ such that $\mathcal{A} \neq \varnothing_{S N}$ and $\mathcal{A} \neq 1_{S N}$. If there exists such a single-valued neutrosophic regular open set $\mathcal{A}=\left(\mathcal{T}_{\mathcal{A}}, \mathcal{I}_{\mathcal{A}}, \mathcal{F}_{\mathcal{A}}\right)$ such that $\mathcal{A} \neq \varnothing_{S N}$ and $\mathcal{A} \neq 1_{S N}$, then K-algebra is said to be a single-valued neutrosophic super disconnected.

Example 5. Let $\mathcal{K}=(G, \cdot \odot, e)$ be a K-algebra, where $G=\left\{e, x, x^{2}, x^{3}, x^{4}, x^{5}, x^{6}, x^{7}, x^{8}\right\}$ is the cyclic group of order 9 and Caley's table for $\odot$ is given in Example 1 We define a SNS as:

$$
\mathcal{A}=\{(e, 0.2,0.3,0.8),(s, 0.1,0.2,0.6)\}
$$
$\mathcal{K}$. here

Let $\chi_{\mathcal{K}}=\left\{\varnothing_{S N}, 1_{S N}, \mathcal{A}\right\}$ be a SNT on $\mathcal{K}$ and let $\mathcal{B}=\{(e, 0.3,0.3,0.8),(s, 0.2,0.2,0.6)\}$ be a SNS in

SNOSS $: \varnothing_{S N}=\{0,0,1\}, 1_{S N}=\{1,1,0\}, \mathcal{A}=\{(e, 0.2,0.3,0.8),(s, 0.1,0.2,0.6)\}$.

SNCSS : $\left(\varnothing_{S N}\right)^{c}=(\{0,0,1\})^{c}=(\{1,1,0\})=1_{S N},\left(1_{S N}\right)^{c}=(\{1,1,0\})^{c}=(\{0,0,1\})=\varnothing_{S N}$,

$(\mathcal{A})^{c}=(\{(e, 0.2,0.3,0.8),(s, 0.1,0.2,0.6)\})^{c}=(\{(e, 0.8,0.3,0.2),(s, 0.6,0.2,0.1)\})=\mathcal{A}^{\prime}($ say $)$. 
Then, closure of $\mathcal{B}$ is the intersection of closed sets which contain $\mathcal{B}$. Therefore,

$$
\mathcal{A}^{\prime}=\mathcal{B}^{\text {Clo }} .
$$

Now, interior of $\mathcal{B}$ is the union of open sets which contain in $\mathcal{B}$. Therefore,

$$
\begin{gathered}
\varnothing_{S N} \cup \mathcal{A}=\mathcal{A} \\
\mathcal{A}=\mathcal{B}^{I n t} .
\end{gathered}
$$

Note that $\left(\mathcal{B}^{C l o}\right)^{C l o}=\mathcal{B}^{C l o}$. Now, if we consider a SNS $\mathcal{A}=\{(e, 0.2,0.3,0.8),(s, 0.1,0.2,0.6)\}$ in a $K$-algebra $\mathcal{K}$ and if $\chi_{\mathcal{K}}=\left\{\varnothing_{S N}, 1_{S N}, \mathcal{A}\right\}$ is a SNT on $\mathcal{K}$. Then, $(\mathcal{A})^{\text {Clo }}=\mathcal{A}$ and $(\mathcal{A})^{\text {Int }}=\mathcal{A}$. Consequently,

$$
\mathcal{A}=\left(\mathcal{A}^{\text {Clo }}\right)^{\text {Int }},
$$

which shows that $\mathcal{A}$ is a $S N$ regular open set in $K$-algebra $\mathcal{K}$. Since $\mathcal{A}$ is a $S N$ regular open set in $\mathcal{K}$ and $\mathcal{A} \neq \varnothing_{S N}, \mathcal{A} \neq 1_{S N}$, then, by Definition $17, K$-algebra $\mathcal{K}$ is a single-valued neutrosophic supper disconnected K-algebra.

Proposition 6. Let $\mathcal{K}$ be a K-algebra and let $\mathcal{A}$ be a SNOS. Then, the following statements are equivalent:

(i) A K-algebra is single-valued neutrosophic super connected.

(ii) $(\mathcal{A})^{\text {Clo }}=1_{S N}$, for each $S N O S \mathcal{A} \neq \varnothing_{S N}$.

(iii) $(\mathcal{A})^{\text {Int }}=\varnothing_{S N}$, for each SNCS $\mathcal{A} \neq 1_{S N}$.

(iv) There do not exist SNOSs $\mathcal{A}, \mathcal{F}$ such that $\mathcal{A} \subseteq \overline{\mathcal{F}}$ and $\mathcal{A} \neq \varnothing_{S N} \neq \mathcal{F}$ in K-algebra $\mathcal{K}$.

Definition 18. Let $(\mathcal{K}, \chi)$ be a SNTS, where $\mathcal{K}$ is a K-algebra. Let $S$ be a collection of SNOSs in $\mathcal{K}$ denoted by $S=\left\{\left(\mathcal{T}_{\mathcal{A}_{j}}, \mathcal{I}_{\mathcal{A}_{j}}, \mathcal{F}_{\mathcal{A}_{j}}\right): j \in J\right\}$. Let $\mathcal{A}$ be a SNOS in $\mathcal{K}$. Then, $S$ is called a single-valued neutrosophic open covering of $\mathcal{A}$ if $\mathcal{A} \subseteq \cup S$.

Definition 19. Let $\mathcal{K}$ be a $K$-algebra and $(\mathcal{K}, \chi)$ be a SNTS. Let $L$ be a finite sub-collection of $S$. If $L$ is also a single-valued neutrosophic open covering of $\mathcal{A}$, then it is called a finite sub-covering of $S$ and $\mathcal{A}$ is called single-valued neutrosophic compact if each single-valued neutrosophic open covering $S$ of $\mathcal{A}$ has a finite sub-cover. Then,$(\mathcal{K}, \chi)$ is called compact K-algebra.

Remark 3. If either $\mathcal{K}$ is a finite $K$-algebra or $\chi$ is a finite topology on $\mathcal{K}$, i.e., consists of finite number of single-valued neutrosophic subsets of $\mathcal{K}$, then the $\operatorname{SNT}(\mathcal{K}, \chi)$ is a single-valued neutrosophic compact topological space.

Proposition 7. Let $\left(\mathcal{K}_{1}, \chi_{1}\right)$ and $\left(\mathcal{K}_{2}, \chi_{2}\right)$ be two SNTSs and $f$ be a single-valued neutrosophic continuous mapping from $\mathcal{K}_{1}$ into $\mathcal{K}_{2}$. Let $\mathcal{A}$ be a SNS in $\left(\mathcal{K}_{1}, \chi_{1}\right)$. If $\mathcal{A}$ is single-valued neutrosophic compact in $\left(\mathcal{K}_{1}, \chi_{1}\right)$, then $f(\mathcal{A})$ is single-valued neutrosophic compact in $\left(\mathcal{K}_{2}, \chi_{2}\right)$.

Proof. Let $f:\left(\mathcal{K}_{1}, \chi_{1}\right) \rightarrow\left(\mathcal{K}_{2}, \chi_{2}\right)$ be a single-valued neutrosophic continuous function. Let $\hat{S}=\left(f^{-1}\left(\mathcal{A}_{j}: j \in J\right)\right)$ be a single-valued neutrosophic open covering of $\mathcal{A}$ since $\mathcal{A}$ be a SNS in $\left(\mathcal{K}_{1}, \chi_{1}\right)$. Let $L=\left(\mathcal{A}_{j}: j \in J\right)$ be a single-valued neutrosophic open covering of $f(\mathcal{A})$. Since $\mathcal{A}$ is compact, then there exists a single-valued neutrosophic finite sub-cover $\bigcup_{j=1}^{n} f^{-1}\left(\mathcal{A}_{j}\right)$ such that

$$
\mathcal{A} \subseteq \bigcup_{j=1}^{n} f^{-1}\left(\mathcal{A}_{j}\right)
$$

We have to prove that there also exists a finite sub-cover of $L$ for $f(\mathcal{A})$ such that 


$$
f(\mathcal{A}) \subseteq \bigcup_{j=1}^{n}\left(\mathcal{A}_{j}\right)
$$

Now,

$$
\begin{aligned}
\mathcal{A} & \subseteq \bigcup_{j=1}^{n} f^{-1}\left(\mathcal{A}_{j}\right) \\
f(\mathcal{A}) & \subseteq f\left(\bigcup_{j=1}^{n} f^{-1}\left(\mathcal{A}_{j}\right)\right) \\
f(\mathcal{A}) & \subseteq \bigcup_{j=1}^{n}\left(f\left(f^{-1}\left(\mathcal{A}_{j}\right)\right)\right) \\
f(\mathcal{A}) & \subseteq \bigcup_{j=1}^{n}\left(\mathcal{A}_{j}\right) .
\end{aligned}
$$

Hence, $f(\mathcal{A})$ is single-valued neutrosophic compact in $\left(\mathcal{K}_{2}, \chi_{2}\right)$.

Definition 20. A single-valued neutrosophic set $\mathcal{A}$ in a K-algebra $\mathcal{K}$ is called a single-valued neutrosophic point if

$$
\begin{aligned}
& \mathcal{T}_{\mathcal{A}}(v)= \begin{cases}\alpha \in(0,1], & \text { if } v=u \\
0, & \text { otherwise, }\end{cases} \\
& \mathcal{I}_{\mathcal{A}}(v)= \begin{cases}\beta \in(0,1], & \text { if } v=u \\
0, & \text { otherwise, }\end{cases} \\
& \mathcal{F}_{\mathcal{A}}(v)= \begin{cases}\gamma \in[0,1), & \text { if } v=u \\
0, & \text { otherwise, }\end{cases}
\end{aligned}
$$

with support $u$ and value $(\alpha, \beta, \gamma)$, denoted by $u(\alpha, \beta, \gamma)$. This single-valued neutrosophic point is said to "belong to" a SNS $\mathcal{A}$, written as $u(\alpha, \beta, \gamma) \in \mathcal{A}$ if $\mathcal{T}_{\mathcal{A}}(u) \geq \alpha, \mathcal{I}_{\mathcal{A}}(u) \geq \beta, \mathcal{F}_{\mathcal{A}}(u) \leq \gamma$ and said to be "quasi-coincident with" a SNS $\mathcal{A}$, written as $u(\alpha, \beta, \gamma)$ q $\mathcal{A}$ if $\mathcal{T}_{\mathcal{A}}(u)+\alpha>1, \mathcal{I}_{\mathcal{A}}(u)+\beta>1, \mathcal{F}_{\mathcal{A}}(u)+\gamma<1$.

Definition 21. Let $\mathcal{K}$ be a K-algebra and let $(\mathcal{K}, \chi)$ be a SNTS. Then, $(\mathcal{K}, \chi)$ is called a single-valued neutrosophic Hausdorff space if and only if, for any two distinct single-valued neutrosophic points $u_{1}, u_{2} \in \mathcal{K}$, there exist SNOSs $\mathcal{B}_{1}=\left(\mathcal{T}_{\mathcal{B}_{1}}, \mathcal{I}_{\mathcal{B}_{1}}, \mathcal{F}_{\mathcal{B}_{1}}\right), \mathcal{B}_{2}=\left(\mathcal{T}_{\mathcal{B}_{2}}, \mathcal{I}_{\mathcal{B}_{2}}, \mathcal{F}_{\mathcal{B}_{2}}\right)$ such that $u_{1} \in \mathcal{B}_{1}, u_{2} \in \mathcal{B}_{2}$, i.e.,

$$
\begin{aligned}
& \mathcal{T}_{\mathcal{B}_{1}}\left(u_{1}\right)=1, \mathcal{I}_{\mathcal{B}_{1}}\left(u_{1}\right)=1, \mathcal{F}_{\mathcal{B}_{1}}\left(u_{1}\right)=0, \\
& \mathcal{T}_{\mathcal{B}_{2}}\left(u_{2}\right)=1, \mathcal{I}_{\mathcal{B}_{2}}\left(u_{2}\right)=1, \mathcal{F}_{\mathcal{B}_{2}}\left(u_{2}\right)=0
\end{aligned}
$$

and satisfy the condition that $\mathcal{B}_{1} \cap \mathcal{B}_{2}=\varnothing_{S N}$. Then, $(\mathcal{K}, \chi)$ is called single-valued neutrosophic Hausdorff space and $\mathrm{K}$-algebra is said to ba a Hausdorff $\mathrm{K}$-algebra. In fact, $(\mathcal{K}, \chi)$ is a Hausdorff K-algebra.

Example 6. Let $\mathcal{K}=(G, \cdot \odot, e)$ be a K-algebra and let $\left(\mathcal{K}, \chi_{\mathcal{K}}\right)$ be a SNTS on $\mathcal{K}$, where $G=\left\{e, x, x^{2}, x^{3}, x^{4}, x^{5}, x^{6}, x^{7}, x^{8}\right\}$ is the cyclic group of order 9 and Caley's table for $\odot$ is given in Example 1 . We define two SNSs as $\mathcal{A}=\{(e, 1,1,0),(s, 0,0,1)\} \cdot \mathcal{B}=\{(e, 0,0,1),(s, 1,1,0)\}$. Consider a single-valued neutrosophic point for $e \in \mathcal{K}$ such that

$$
\begin{aligned}
\mathcal{T}_{\mathcal{A}}(e) & = \begin{cases}0.3, & \text { if } e=u \\
0, & \text { otherwise, }\end{cases} \\
\mathcal{I}_{\mathcal{A}}(e) & = \begin{cases}0.2, & \text { if } e=u \\
0, & \text { otherwise, }\end{cases}
\end{aligned}
$$




$$
\mathcal{F}_{\mathcal{A}}(e)= \begin{cases}0.4, & \text { if } e=u \\ 0, & \text { otherwise } .\end{cases}
$$

Then, $e(0.3,0.2,0.4)$ is a single-valued neutrosophic point with support e and value $(0.3,0.2,0.4)$. This single-valued neutrosophic point belongs to SNS " $A$ " but not SNS " $B$ ".

Now, for all $s \neq e \in \mathcal{K}$

$$
\begin{aligned}
& \mathcal{T}_{\mathcal{B}}(s)= \begin{cases}0.5, & \text { if } s=u \\
0, & \text { otherwise, }\end{cases} \\
& \mathcal{I}_{\mathcal{B}}(s)= \begin{cases}0.4, & \text { if } s=u \\
0, & \text { otherwise, }\end{cases} \\
& \mathcal{F}_{\mathcal{B}}(s)= \begin{cases}0.3, & \text { if } s=u \\
0, & \text { otherwise. }\end{cases}
\end{aligned}
$$

Then, $s(0.5,0.4,0.3)$ is a single-valued neutrosophic point with support $s$ and value $(0.5,0.4,0.3)$. This single-valued neutrosophic point belongs to SNS " $B$ " but not SNS " $A$ ". Thus, $e(0.3,0.2,0.4) \in \mathcal{A}$ and $e(0.3,0.2,0.4) \notin \mathcal{B}, s(0.5,0.4,0.3) \in \mathcal{B}$ and $s(0.5,0.4,0.3) \notin \mathcal{A}$ and $\mathcal{A} \cap \mathcal{B}=\varnothing_{S N}$. Thus, K-algebra is a Hausdorff $K$-algebra and $\left(\mathcal{K}, \chi_{\mathcal{K}}\right)$ is a Hausdorff topological space.

Theorem 5. Let $\left(\mathcal{K}_{1}, \chi_{1}\right),\left(\mathcal{K}_{2}, \chi_{2}\right)$ be two SNTSs. Let $f$ be a single-valued neutrosophic homomorphism from $\left(\mathcal{K}_{1}, \chi_{1}\right)$ into $\left(\mathcal{K}_{2}, \chi_{2}\right)$. Then, $\left(\mathcal{K}_{1}, \chi_{1}\right)$ is a single-valued neutrosophic Hausdorff space if and only if $\left(\mathcal{K}_{2}, \chi_{2}\right)$ is a single-valued neutrosophic Hausdorff K-algebra.

Proof. Let $\left(\mathcal{K}_{1}, \chi_{1}\right),\left(\mathcal{K}_{2}, \chi_{2}\right)$ be two SNTSs. Let $\mathcal{K}_{1}$ be a single-valued neutrosophic Hausdorff space, then, according to the Definition 21, there exist two SNOSs $X$ and $Y$ for two distinct single-valued neutrosophic points $u_{1}, u_{2} \in \chi_{2}$ also $a, b \in \mathcal{K}_{1}(a \neq b)$ such that $X \cap Y=\varnothing_{S N}$.

Now, for $w \in \mathcal{K}_{1}$, consider $\left(f^{-1}\left(u_{1}\right)\right)(w)=u_{1}\left(f^{-1}(w)\right)$, where $u_{1}\left(f^{-1}(w)\right)=s \in(0,1]$ if $w=f^{-1}(a)$, otherwise 0. That is, $\left(f^{-1}\left(u_{1}\right)\right)(w)=\left(\left(f^{-1}(u)\right)_{1}(w)\right)$. Therefore, we have $f^{-1}\left(u_{1}\right)=\left(f^{-1}(u)\right)_{1}$. Similarly, $f^{-1}\left(u_{2}\right)=\left(f^{-1}(u)\right)_{2}$. Now, since $f^{-1}$ is a single-valued neutrosophic continuous mapping from $\mathcal{K}_{2}$ into $\mathcal{K}_{1}$, there exist two SNOSs $f(X)$ and $f(Y)$ of $u_{1}$ and $u_{2}$, respectively, such that $f(X) \cap f(Y)=f\left(\varnothing_{S N}\right)=\varnothing_{S N}$. This implies that $\mathcal{K}_{2}$ is a single-valued neutrosophic Hausdorff $K$-algebra. The converse part can be proved similarly.

Theorem 6. Let $f$ be a single-valued neutrosophic continuous function which is both one-one and onto, where $f$ is a mapping from a single-valued neutrosophic compact K-algebra $\mathcal{K}_{1}$ into a single-valued neutrosophic Hausdorff K-algebra $\mathcal{K}_{2}$. Then, $f$ is a homomorphism.

Proof. Let $f: \mathcal{K}_{1} \rightarrow \mathcal{K}_{2}$ be a single-valued neutrosophic continuous bijective function from single-valued neutrosophic compact $K$-algebra $\mathcal{K}_{1}$ into a single-valued neutrosophic Hausdorff $K$-algebra $\mathcal{K}_{2}$. Since $f$ is a single-valued neutrosophic continuous mapping from $\mathcal{K}_{1}$ into $\mathcal{K}_{2}, f$ is a homomorphism. Since $f$ is bijective, we only prove that $f$ is single-valued neutrosophic closed. Let $\mathcal{D}=\left(\mathcal{T}_{\mathcal{D}}, \mathcal{I}_{\mathcal{D}}, \mathcal{F}_{\mathcal{D}}\right)$ be a single-valued neutrosophic closed in $\mathcal{K}_{1}$. If $\mathcal{D}=\varnothing_{S N}$ is single-valued neutrosophic closed in $\mathcal{K}_{1}$, then $f(\mathcal{D})=\varnothing_{S N}$ is single-valued neutrosophic closed in $\mathcal{K}_{2}$. However, if $\mathcal{D} \neq \varnothing_{S N}$, then $\mathcal{D}$ will be a single-valued neutrosophic compact, being subset of a single-valued neutrosophic compact $K$-algebra. Then, $f(\mathcal{D})$, being single-valued neutrosophic continuous image of a single-valued neutrosophic compact $K$-algebra, is also single-valued neutrosophic compact. Therefore, $\mathcal{K}_{2}$ is closed, which implies that mapping $f$ is closed. Thus, $f$ is a homomorphism. 


\section{Conclusions}

Non-classical logic is considered as a powerful tool for inspecting uncertainty and indeterminacy found in real world problems. Being a great extension of classical logic, neutrosophic set theory is considered as a useful mathematical tool to cope up with uncertainties in science, technology, and computer science. We have used this mathematical model with a topological structure to investigate the uncertainty in $K$-algebras. We have introduced the notion of single-valued neutrosophic topological $\mathrm{K}$-algebras and presented certain concepts, including continuous function between two topological on $K$-algebras, relatively continuous function and homomorphism. We have investigated the image and pre-image of single-valued neutrosophic topological $K$-algebras under this homomorphism. We have proposed some conclusive concepts, including single-valued neutrosophic compact $K$-algebras and single-valued neutrosophic Hausdorff $K$-algebras. We plan to extend our study to: (i) single-valued neutrosophic soft topological $K$-algebras; and (ii) bipolar neutrosophic soft topological $K$-algebras.

For other notations and terminologies, readers are referred to [21-26].

Author Contributions: M.A., H.G., F.S. and S.B. conceived of and designed the experiments. M.A. and H.G. wrote the paper

Acknowledgments: The author is highly thankful to anonymous referees for their valuable comments and suggestions for improving the paper.

Conflicts of Interest: The authors declare that they have no competing interests.

\section{References}

1. Dar, K.H.; Akram, M. On a K-algebra built on a group. Southeast Asian Bull. Math. 2005, 29, 41-49.

2. Dar, K.H.; Akram, M. Characterization of a K(G)-algebra by self maps. Southeast Asian Bull. Math. 2004, 28, 601-610.

3. Dar, K.H.; Akram, M. On K-homomorphisms of K-algebras. Int. Math. Forum 2007, 46, 2283-2293. [CrossRef]

4. Akram, M.; Dar, K.H.; Jun, Y.B.; Roh, E.H. Fuzzy structures of K(G)-algebra. Southeast Asian Bull. Math. 2007, 31, 625-637.

5. Akram, M.; Dar, K.H. Generalized Fuzzy K-Algebras; VDM Verlag: Saarbrücken, Gernamy, 2010; p. 288, ISBN 978-3-639-27095-2.

6. Smarandache, F. Neutrosophy Neutrosophic Probability, Set, and Logic; Amer Res Press: Rehoboth, MA, USA, 1998.

7. Atanassov, K. Intuitionistic fuzzy sets. Fuzzy Sets Syst. 1986, 20, 87-96. [CrossRef]

8. Wang, H.; Smarandache, F.; Zhang, Y.Q.; Sunderraman, R. Single valued neutrosophic sets. Multispace Multistruct 2010, 4, 410-413.

9. Agboola, A.A.A.; Davvaz, B. Introduction to neutrosophic BCI/BCK-algebras. Int. J. Math. Math. Sci. 2015, 6. [CrossRef]

10. June, Y.B. Neutrosophic subalgebras of several types in BCK/BCI-algebras. Annl. Fuzzy Math. Inform. 2017, 14, 75-86.

11. June, Y.B.; Kim, S.J.; Smarandache, F. Interval neutrosophic sets with applications in BCK/BCI-algebra. Axioms 2018, 7, 23. [CrossRef]

12. Jun, Y.B.; Smarandache, F.; Song, S.Z.; Khan, M. Neutrosophic positive implicative $N$-ideals in $B C K$-algebras. Axioms 2018, 7, 3. [CrossRef]

13. Chang, C.L. Fuzzy topological spaces. J. Math. Anal. Appl. 1968, 24, 182-190. [CrossRef]

14. Lowen, R. Fuzzy topological spaces and fuzzy compactness. J. Math. Anal. Appl. 1976, 56, 621-633. [CrossRef]

15. Pu, P.M.; Liu, Y.M. Fuzzy topology, I. Neighbourhood structure of a fuzzy point and Moore-Smith convergence. J. Math. Anal. Appl. 1980, 76, 571-599.

16. Chattopadhyay, K.C.; Samanta, S.K. Fuzzy topology: Fuzzy closure operator, fuzzy compactness and fuzzy connectedness. Fuzzy Sets Syst. 1993, 54, 207-212. [CrossRef]

17. Coker, D. An introduction to intuitionistic fuzzy topological spaces. Fuzzy Sets Syst. 1997, 88, 81-89. [CrossRef] 
18. Salama, A.A.; Alblowi, S.A. Neutrosophic set and neutrosophic topological spaces. IOSR-JM 2012, 3, 31-35. [CrossRef]

19. Akram, M.; Dar, K.H. On fuzzy topological K-algebras. Int. Math. Forum 2006, 23, 1113-1124. [CrossRef]

20. Akram, M.; Dar, K.H. Intuitionistic fuzzy topological K-algebras. J. Fuzzy Math. 2009, 17, 19-34.

21. Lupianez, F.G. Hausdorffness in intuitionistic fuzzy topological spaces. Mathw. Soft Comput. 2003, 10, 17-22.

22. Hanafy, I.M. Completely continuous functions in intuitionistic fuzzy topological spaces. Czechoslovak Math. J. 2003, 53, 793-803. [CrossRef]

23. Jun, Y.B.; Song, S.Z.; Smarandache, F.; Bordbar, H. Neutrosophic quadruple BCK/BCI-algebras. Axioms 2018, 7, 41. [CrossRef]

24. Elias, J.; Rossi, M.E. The structure of the inverse system of Gorenstein K-algebras. Adv. Math. 2017, 314, 306-327. [CrossRef]

25. Masuti, S.K.; Tozzo, L. The structure of the inverse system of level K-algebras. Collect. Math. 2017, 1-27. [CrossRef]

26. Borzooei, R.; Zhang, X.; Smarandache, F.; Jun, Y. Commutative generalized neutrosophic ideals in BCK-algebras. Symmetry 2018, 10, 350. [CrossRef]

(C) 2018 by the authors. Licensee MDPI, Basel, Switzerland. This article is an open access article distributed under the terms and conditions of the Creative Commons Attribution (CC BY) license (http://creativecommons.org/licenses/by/4.0/). 